\title{
Sachregister zu Band 76
}

Die fett gedruckten Zahlen bezeichnen Eigenarbeiten. Bb. = Buchbesprechung.

A

Ablatio retinae 171

erbliche 397

Gefäßschatten als neues Symptom der - 406

geheilt mittels Goninscher Operation 410

Rezidiv einer -, durch Operation nach Larsson geheilt 405

Akkommodation 109 Albinismus bei Negern 390 Altersbrillen, wann werden -

gekauft ? 217 Amýlnitrit, Behandlung der Au-

genhintergrundserkrankungen mit

101 Anatomie 166ff. (Ber.)Angioide Streifenbildung 208Angiom der Chorioidea

221 Angiomatosis retinae 173, 332Aniridie 187

- Vererbung der angeborenen -

389 Anisometrie und bilaterale Sym-

metrie 109 Ankyloblepharon, familiäres 388 Anophthalmus, angeborener 208 - erblicher 390

Aphakie, spontane 408

angeborene familiäre 390 Arachnodaktylie, familiäre 389

und Ektopia lentis 190 Argolaval 98 Arsenik-Keratitis 333 Arterienschlinge, in den Glas-

körper vorragende - 404 Arteriosklerose des Auges 173 Astigmatismus, Bestimmung des

$-\quad 380$

- $\quad$ Vererbung des - 393, 394Astigmie, Pathogenese der - 117Aubert-Förstersches Phänomen 319 Augenärztliche Eingriffe 336

(Bb.) Augenbraue, Entwicklung der -

166 Augendruck s. Druck Augengeschwülste, chirurgische

Radiumbehandlung bösartiger -

97 Augenhintergrund, leukämische

Veränderungen des - 358 Augenhintergrundsphotogra-

phien, stereoskopische -108

Augenlider s. Lider Augenmuskeln, erbliche Affektio-

nen der - 388 Augenoperationen und Diabetes

1a5 Augenspalte, Entstehungsweise

und Verschluß der fötalen - 183 Augentropfen und Augensalben,

rationale Anwendung der -411

B

Bacterium fluorescens, Infek-

tion der Bindehaut mit - 117 Bacterium granulosis, pathologi-

sche Bedeutung des - Ill Bakterienkulturen auf Linsen-

nährboden 116 Bewegungssehen 319 Berufswahl und Auge 336 (Bb.) Bifokalbrille 376

Bindegewebige Fasern in den 
Augenlidern, Entwicklung der -

182 Bindehaut s. Conjunctiva Binokuläres Sehen 312 Bjerrumtafel 326 Blepharochalasis, Anatomie der

- $\quad$ 166Blepharospasmus als Frühsym-

ptom der Tabes dorsalis $121-\cdot$ hystericus traumaticus 206

Behandlung des essentiellen - 94

Elektrokoagulation bei schwerem

- 337, 406Blutansammlung, scheibenförmi-

ge - auf der Hornhautrückfläche nach Kataraktextraktion 92

Blutf arbstof färbung, Ursachen der differenten - von Hornhaut, Linse und Glaskörper 209

Brillenkunde 197

Buphthalmus mit Naevus flam-meus 185

C Chiasma opticum, topographische

Anatomie des -- 404 Cholesterinkristalle in der Linse

94

- $\quad$ in der Vorderkammer 411Chorioidea, Angiom der - 22 $\tau-$ - Sarkom der - mit Durchbruch

in die Orbita $92-\cdot$ geheilte postoperative Spätab-lösung der - 207

Sachregister

Chorioidea, Schaffungvonflächen-haften Verlötungen der Netzhaut mit der - durch Endothermie 90

Chorioretinitis, atypische Form der - bei angeborener Lues 328

Chromotropie 176

Conjunctiva, Infektion der - mit Bacterium fluorescens 117

Pigmentierung der - bei Vitamin-A-Mangelerkrankung 412

Tuberkulose der -147

Tumoren der -- 168

Bindehautdeckung bei Hornhaut-geschwüren 213

Bindehautdeckung bei der Star-operation 300

Cornea, normale und abnorme Ent-wicklung der - 180

Regeneration des Epithels der - beim Kaninchen 324

Form der vorderen Fläche der

- $\quad 372$

senkrecht ovale Form der - 187

pathologische Anatomie der -169

Haardruckempfindlichkeit der - 321

familiäre degenerative Erkran-kungen der - 91

erbliche Affektionen der - 394

Krebs der - von einem Ptery-giumkopf ausgehend 112

neuropathische Erkrankungen der

$-\quad 114$

- $\quad$ Astigmatismus und Brechung

380, 394

- Dystrophie, Vererbung der wir-belförmigen - -395

-Scheibengrößen 381, 394

-Trübungen, angeborene 186

-Überpflanzung, vollständige, durchgreifende - 215 
Cornu cutaneum des Augenlids 166

D

Dakryozystitis, erbliche familiäre 388

Dakryozystoblennorrhoe, erbliche 191

Diabetesund Augenoperationen 105

Dioptrik 369 (Ber.)

Dreifokusglas 376

Druck, intraokulärer 324

Dunkeladaptation 314

Dysostosis cranio-facialis, erbliche 398

Dystrophia epithelialis cor' neae 169

E

Echinokokkusdes Glaskörpers 172 Eidetische Fähigkeiten 318

zu Band 76. 42I

Eiweißgehalt im Kammerwasser, medikamentöse Beeinflussung des

- $\quad$ 116Ektopia lentis 190

- Vererbung der -389

Ekthyma gangraenosum ca-checticorum mit parenchyma-töser Keratitis auf nichtsyphili-tischer Basis 122

Ekzem und Katarakt 326

Elektrokoagulation bei schwe-rem Blepharospasmus 337, 406

Endothermie, Schaffung von flä-chenhaften Verlötungen der Netzhaut mit der Aderhaut durch 90

Entwicklungsgeschichte 178

Eriziskop 375

Exophthalmus pulsans, konser-vative Behandlung des a-219

F

Farben, Wirkung der - 320 Farbenblindheit 308

- $\quad$ erbliche 396Farbensehen 306Fazialiskrampf, hereditärer 389Fazialislähmung, familiäre

rezi-

divierende - 389 Fleischer-Kayserscher Ring als

Frühsymptom der Wilsonschen

Krankheit 327 Flügelfelloperation, Replantatio

pterygii als Methode der - 157 Formensehen 313 Formwahrnehmungen 318 Fremdkörper, das weitere Schick-

sal nicht entfernter intraokulärer

- $\quad 96$

Fuchssche Heterochromie 187, 188

$\mathrm{G}$

Gasaustausch der Hornhaut mit der Luft 324

GaucherscheKrankheit und Seh-organ 129

Gefäßschatten als neues Ablatio-symptom 406

Gefäßsystem des Auges, Entwicklungsgeschichte des - 180

- $\quad$ Anomalien des - 188Gehirntumoren ohne Stauungs-

papille 222 Geschichte der Ophthalmologie 195 Gelbkreuzgas, Verletzungen mit

- $\quad$ 326Gestaltwahrnehmungen 318Gewebe des Auges, Ernährung und 
Stoffwechsel der - 223 Gewebspharmakologie des Auges 209

422

Sachregister zu Band 76.

Glaskörper, pathologische Ana-tomie des - 77

- $\quad$ Echinokokkus des - 172Glaskörperstränge mit zirkum-

papillärer und mit präpapillärer

Membran 208, 209 Glaskörpertrübung, beiderseitige

frische ringförmige - 199 Glasmacherstar 325 Glaukom 93

Vererbung des -391

experimentelles - bei Hunden

175

ohne Hóchdruck 208

mit Naevus flammeus 185

und Netzhautzirkulation $201 \bullet-$ myopisches 220

Behandlung des chronischen -346

chirurgische Behandlung des chronischen - 95

Gliom, Histologie des -117

- $\quad$ Vererbung des -391

- $\quad$ der Netzhaut 174, 175Goldtherapie der Lues 415Goninsche Operation bei Ablatio

retinae 410 Gonorrhoe, Neuritis retrobulbaris

infolge von - 409 Granulationsgeschwülste der

Lider 166 Größenwahrnehmungen 318 Grubenbildung der Papille 185

$\mathrm{H}$

Haardruckempfindlichkeit der

Hornhaut 321 Haftgläser 114, 370, 371 Handarbeiten und Sehschärfe 113,

287 Hautkrankheiten, Beziehungen

der - zu Krankheiten des Seh-

organs 121 Hemianopische Pupillenstarre

208 Hemianopsie, anatomische Verän-

derungen bei binasaler - $174 \cdot-$ topische Diagnostik der - 241 Hemiatrophia faciei 206

Hemikinesimeter 108 Herpes simplex 332 Heterochromia iridis 390

- $\quad$ Fuchssche 187, 188Histologie, Handbuch der - 335

(Bb). Hornhaut s. Cornea Hydrophthalmus 391

- und Naevus flammeus 198Hypermetropie, erbliche 393Hypertelorismus 387Hypertonie, medikamentöse Be-

einflussung der reaktiven -115

I

Idiotic, familiäre amaurotische 225,

330 (Bb.), 396 Infiltration, Beziehungen der -

zur Narbenbildung bei Trachom

329 Iridozyklitis, Ätiologie endogener

$-\quad 119$

- $\quad$ tuberkulöse bei Lupus vulgaris 112Iris, Pigmentierungsverhältnisse der

$-\quad 194$ 
- $\quad$ Sarkom der - 170Irispigment bei den Japanern 183Iriszyste, traumatische - mit kristallinischem Inhalt 408

$\mathrm{K}$

Kammerwasser, medikamentöse Beeinflussung des Eiweißgehaltes im - 116

Kammerwinkel, Entwicklung des

- $\quad 179$

Katarakt, rasch entstandene -207

Vererbung der - 189, 190, 395

pulverulenta 73

Glasmacherstar 325

und Ekzem 326

-. electrica durch Starkstrom 213 Kataraktbildung, physikochemischer Mechanismus der - 106 Kataraktoperation, Ausziehung

des Altersstars in der Kapsel 326

- $\quad$ Bindehautdeckung bei der - 303

- $\quad$ scheibenförmige Blutansammlungauf der Hornhautrückfläche nach

$-\quad 92$

- $\quad$ spontane Verdünnung der Skleraals Störung bei der - 365

Keratitis, Arsen- - 333

follicularis spinulosa decalvans, familiäre 388

knötchenförmige familiäre 395

parenchymatosa mit Ekthyma gangraenosum cachecticorum auf nichtsyphilitischer Basis 122 punctata superficialis, diagnosti-scher Wert der - des Kaninchens 114

Rosacea- - bei fehlender Oder geringer Hautrosacea 218

Keratokonus, familiärer 395 Keratomalazie, Sensibilität bei -

321 Keratoplastik 217

- $\quad$ totale 409Kleinhirnbrückentumor, Verän-

derungen der Sehnerven bei - 175

Knochenbildung am Auge 176

Kobaltlampe 374

Kokaineinlagen in die Nase bei

Tränensackphlegmonen und aku-

ten Schwellungen der Lider 283

Sachregister zu Band 76.

423

Kolobome 185

- $\quad$ Vererbung kolobomatöser Affek-tionen 389

Komplementbindungsreaktion auf Tuberkulose, Bewertung der

- $\quad$ bei inneren' Augenerkrankun-gen 93

Kopfschmerzen, Augensymptome

bei myalgischen - 199 Krukenbergsche Pigmentspin-

del 187, 391

L Larocain 210 Laurence - Biedlsche Krank-

heit, Leberfunktion bei - 156 Leberfunktion bei Laurence-

Biedlscher Krankheit 156 Lebersche Erkránkung, anato- 
mische Befunde bei - 175 Lenticonus posterior 278

- $\quad$ Spaltlampenbefund bei - 107Leseschwäche 320Leukämische Fundusverände-

rungen 358 Leukome, angeborenedoppelseitige,

- der Hornhaut 186Lichtsinn 305

Lichtmischung, Gesetz der - 307 Lider, pathologische Anatomie der

- 166

Mißbildungen der -- 191

Pars orbitalis palp. sup. und ihre Bedeutung für das individuelle Auge 61

erbliche Affektionen der - 386

Behandlung akuter Schwellungen der - mit Kokaineinlagen in die Nase 283

Lidschluß, Epithelveränderungen bei der Lösung des fötalen - 182

Limbuspigmentierung, abnorme 200

Li use, Anatomie der - 172

Entwicklung der -181

Brechungsindex der fötalen -183

pathologische Anatomie der - 73

erbliche Anomalien der - 395

Abschnürung der - vom Ekto-derm 182

Linsennährboden, Bakterienkul-

turen auf - 116 Li nsenluxation, erbliche 389, 390 Lipoide 223 Lipoidstoffwechsel, Krankheiten

durch Störung des - in Beziehung

zum Sehorgan 129 Liquor cerebrospinalis 335 (Bb.)

- $\quad$ moderne Luesdiagnostik mit be-sonderer Berücksichtigung des- 1

Lokalisations-Perimeter 108 Liimbaldruck, Steigerung des - bei Druck auf den Bulbus 322

Lupus vulgaris, Iridocyclitis tu-berculosa bei - 112

$\mathrm{M}$

Macula lutea, angeborene Ver-

änderung der - 100 Makropsie, Erzeugung von - 312 Medikamente, Resorption der -

aus Salben 118 Mikuliczsche Krankheit 167 Megalócornea 187

einseitige 90

und Katàrakt 395 Melanoblastom der Iris 329 Melanosis conjunctivae 208 Melanosis bulbi 107

Membran, zirkumpapilläre und prä-

papilläre - mit Glaskörpersträn-

gen 208, 209 Mikrophakie, familiäre 190 Mikrophthalmus, familiärer 390

- $\quad$ familiärer -- mit Zwergwuchs 185

- $\quad$ mit Katàrakt und Iriskolobom200

Mikropsie, Erzeugung von - 312 Mißbildungen 184ft. Myopie, Entstehung der - 377ft., 392, 393

Refraktions- - 372, 379

glaukomatöse 220

und Sehnervenkolobom 186

N Nachbilder, Latenzzeit von -- 305 Naevus flammeus und Hydr-ophthalmus 198

bei Buphthalmus und bei Glau-kom 185

Nebenhöhlen, Topographie der - mit Rücksicht auf die Erkran-kungen des Sehnerven 109

Ñervus opticus, erbliche Anomalien des - 396

- $\quad$-Atrophie, erbliche 398Netzhaut s. RetinaNetzhautablösung s. Ablatio

retinae 
Neurinom, intra-retrobulbäres - 112

Neuritis optici tonsillären Ur-sprungs 100

Neuritis retrobulbaris mit Herpes simplex 332

- $\quad$ gonorrhoischen Ursprungs 409Niemann-Picksche Krankheit

und Sehorgan 129 Nystagmus der Albinos 188

- $\quad$ erblicher 192, 389

- $\quad$ Krankheitsbild des - der Berg-leute in England und Deutschlandund sein Einfluß auf die

Erwerbs-fähigkeit 29

424

Sachregister

zu Band 76.

\section{0}

Oculomotoriuslähmung, rezidi-vierend erbliche - mit Migräne .. 388 Ödem, angeborenes angioneuroti-

sches - der Lider 166 Okulo-kardiale Reflexe 322 Ophthalmia sympathica 171 Ophthalmoplegie, erbliche 388 - Handbuch der - 124 (Bb.) Optik, physikalische 369 (Ber.) -· physiologische 303 (Ber.) - psychologische 318 (Ber.) Organextrakte, Wirkung der -

auf das Froschauge 115 Osteom der Orbital wand 403

$P$

Palpebralzyste, anatomische Be-

funde bei - 219 Papillen, ungewöhnlich große -

189 - Grubenbildung der - 185 Papilloretinitis tuberculosa,

Retinitis proliferans als Folge

einer Bartelschen - 96 Paralyse, progressive, normale

Pupillenreaktion bei - 415 Parinaudsche Conjunctivitis 90 Pathologische Anatomie $166 \mathrm{ff}$.

(Ber.) - - Handbuch der speziellen - 335

(Bb.) Pemphigus conj unctivae, Impf-

versuche mit - 107 Peridermbildung 181 Perimeter, Lokalisations- - 108 Perimetrie 308 Perkain

als Oberflächenanästheti-

kum 157 Pfeilschattenprobe 375 Phlyktänulose, Ätiologie der -

120 Photoskop 373

Physiologische Optik 303 (Ber.) Pigment, Entstehung des melanoti-

schen - 183 Pigmentierung der Bindehaut bei

der Vitamin-A-Mangelerkrankung

412 Pigmentierung, Anomalien der

$-\quad 39^{\circ}$

Pigmentstreif enerkrankungder

Netzhaut 397 Pigmentspindel, Krukenbergsche

- $\quad$ 187, 391Plasmom und Trachom 414Plica semilunaris, abnorme Ent-

wicklung der - 388 Pneumokokkentypen bei Augen-

erkrankungen 414 Primatenauge 323

Pseudogliom 188, 189

- $\quad$ durch Tuberkulose 332Pseudosklerosering der Cornea

169 Psychologische Optik 318 (Ber.) Pterygium, Replantatio pterygii

als Methode der Flügelfellopera- 
tion 157 Pterygiumkopf, von einem -

ausgehendes Hornhautkarzinom

112 Ptosis, Vererbung angeborener -

389 Pupillenreaktion, normale - bei

progressiver Paralyse 415 Pupillensperre, physiologische -

118 Pupillenstarre, hemianopische 208

..Q

QuinckeschesÖdem, Stammbaum vom - 398

$\mathrm{R}$

Radiumbehandlung, chirurgische

- bösartiger Tumoren 97Raumsinn 311Raumvorstellung 312Raumwahrnehmungen

318Rechenstab, optischer 210Recklinghausensche Krank-

heit, ophthalmolog. Beziehungen der -- 112

- $\quad$ und Affektionen der Lider 386,387Reflexe 321Refraktionsanomalien, Klinik

und Entstehung der - 377, 391 Ref raktionsbestimmung, Me-

thoden der - 369 (Ber.) Refraktionsmyopie 372, 379 Reizkörpertherapie, Wirkungder

- $\quad$ auf das Auge 170Replantatio pterygii als Methode der Flügelfelloperation 157

Retina, Anatomie der - 173

Anomalien der - 189

erbliche Anomalien der -- 396

- $\quad$ Zirkulation der - und Glaukom201

-. Schaffung von flächenhaften Ver-

lötungen der - mit der Aderhaut

durch Endothermie 90

Retinitis exsudativa Coats 332

Retinitis pigmentosa, erbliche

397

- $\quad$ sine pigmento 208Retinitis proliferans als Folge

einer Bartelschen Papilloretinitis tuberculosa 96 Retinitis pseudoalbuminurica

97 Retrolentaler Raum 198

Sachregister zu Band $76 . \quad 425$

Rhinostomie plus Tränensackex-

stirpation 103 Röntgenschädigung des Auges

174 Rosacea-Keratitis bei fehlender

oder geringer Hautrosacea 218

$\mathrm{S}$

S alb en, Resorption von Medika-

menten aus - 118 Sarkom der Iris 170

- Zusammenhang zwischen angeb-lichem Unfall und

Entstehungbzw.Verschlimmerungeines-123

Schallreiz und Sehschärfe 311 Schüller-Christiansche Krank-

heit und Sehorgan 129 Scheinbewegungen 313 Schwellenbestimmungen 315 Sehbahnen 173

Sehnerv s. Nervus opticus Sehschärfe 309, 381

- $\quad$ im Gesichtsfeld 118

- $\quad$ und Handarbeiten 113, 287Sehschwache, Unterricht der-383Sella turcica, Größe der -

194Sensibilität 321 Sicherheitsgläser, optisch wirk- 
same $-\cdot 238$ Simulationspröfung, Spiegelseh-

proben zur - 383 Skiaskopie 373 Skisportverletzungen des Auges

334 Sklera, blaue 187

- $\quad$ Vererbung der blauen - 395

- $\quad$ spontane Verdünnung der - alsStörung bei der Staroperation 365

Sklerektomie 346 Skleritis, schwere sulzige - 83 Sklero-Keratometer 371 Skleroperikeratitis 169

Solluxlampe 99 Spiegelsehproben zur Simula-

tionsprüfung 383 Staphylom, angeborenes - der

Hornhaut 186 Starkstrom, Katarakta electrica

durch - 213 Stenopäische Brillen 376 Stereo-Röntgenaufnahmen 210 Streifenbildung, angioide

208 Streptokokkenabszesse im Seh-

nerven 175 Supercilium und Pars orbitalis

palp. sup. und ihre Bedeutung

fur das individuelle Auge 61 Sympathikuslähmung, Rezidi-

vieren von Hornhautgeschwüren

infolge trophischer Störung bei -

206 Synästhesien 318 Synchisis scintillans 77

Syphilis, moderne - -Diagnostik mit besonderer Berücksichtigung des Liquor cerebrospinalis 1

- $\quad$ Goldtherapie der -415

$\mathrm{T}$

Tabes dorsalis, Blepharospasmus als Frühsymptom der -匹 121

Trachom, pathologische Anatomie des - 167

Statistik des - in Ostpreußen 293

ätiologisch - marphologische For-schungsergebnisse überdâs - 416 (Bb.)

Beziehungen der Infiltration zur Narbenbildung bei --329

pathologische Bedeutung des Bacterium granulosis Ill

und Plasmom 414

Organisation zur Bekämpfung des

- 419

- $\quad$ Kurpfuscherische Behandlung des

- $\quad$ 328Tränenkanälchen, Entwicklung

der - bei den Japanern 184 Tränensackexstirpation plus

Rhinostomie 103 Tränensackphlegmone, Behandlung der - mit Kokaineinlagen

in die Nase 283 Tuberkelzellen, Nachweis der -

bei Uveitis durch Kultur aus dem

Gewebe 407 Tuberkulöse Augenkrankhei-

ten, spezifische Behandlung der

113

Tuberkulöse der Bindehaut 147

- $\quad$ Pseudogliom durch - 332Turmschädelin zwei Generationen

398 Typhusimpf ung in der Augenheil-

kunde 99

ü Ulcus corneae, Rezidive von -

infolge trophischer Störung bei

Sympathikuslähmung 206

- $\quad$ Bindehautdeckung bei - 213Unfallkunde 122, 334Universitäts-Augenklinik in 
Berlin, ein Arbeitstag an der - vor 50 Jahren 212 Uveitis dentalen Ursprungs 409

- $\quad$ Nachweis von Tuberkelzellen bei -durch Kultur aus dem Gewebe 407

$\mathrm{V}$

Vena centralis, Thrombose der-

174 Vererbung und Auge 386ff. Versicherungskunde 122, 334 Vitamin-A-Mangelerkrankung, Pigmentierung der Conjunctiva

bei -412

426

Namenregister zu Band 76.

W

Wärmestrahlungsmessungen an

der Hornhaut 324 Wilsonsche Krankheit mit Fleischer-Kaysersçhem Ring als Früh-symptom 327

Wolfram-Titan-Bogenlampe, Photoaktivität und therapeutische Anwendung der - 416 (Bb.) X

Xanthelasma, Vererbungdes - 388 Xanthomatose und Sehorgan 129 - mit Defekten in den

Schädel-knochen 224

Xeroderma pigmentosum, fami-

liäres 391 Xerosis, Sensibilität bei - 321

Z

Zähne, Uveitis dentalen Ursprungs

409 Ziliarfortsätze, Entwicklungsaiao-

malie der - $188 \mathrm{Z}$ i 1 i e, abnormes Wachstum einer-191 Zyklodialyse, Erfolge der - .102

Zyklopie 184 Zylinderskiaskopie 373

Namenregister zu Banò 76.

Ad a my k 166 Albrich 99, 119 Alferow 182 Allen 305 Allport 312 Aloigi 322 Altenburger 315

Ammon 305 Antonibon 388 Apin 374 Arkin 312 Asmus 374 Aszalós 97, 99

B

Bader 195 Bailey 387 Balás 117, 124 Balcarek 210 Ballantyne 185 Banister 311 Bartels 29, 378

Bartok 100 Bauer 187 Baurmann 174 Bednarsky 196 Bell 187 Bender 208, 219 Berg 372 Best

310

Bierens de Haan 308 Birch-Hirschfeld

167, 329 Bistis 188 Bjorkman 314 Blackburn 311 v. Blaskovics 410 Blatt 161 Blumenthal 93

Böck 200 Boenheim 156 Borel 309 Borochovic 185 Boros 97, 99 Borsarelli 315

Botteri 188 Bourdier 307 Bran a 409 Broer 320 Brooser 101 Brown 319 Bruckner 124 Brunswik

318 Bruusgard 332 Burdon-Cooper 416 Burzlaff 307 Butterfield 222

C Capdehourat 322 Cardell 187 Clausen 210, 211, 214,

217, 219 Cockayne 386 Cohn 195

Comberg 198, 200, 325 Cornil 386 Creed 305 Crook 314 v. Csapody 101, 105,

109, 410 v. Csapody-Mócsy

113, 287 Cuccia 310 Curtius 388

$\mathrm{D}$

Dallos 114, 408 Daniels 172, 303 Davenport 387 Davis 192 Debenedetti 322 de Decker 388

Devney 392, 394 Dimmick 315 Ditrói 120 Dollfus 185 Dömsödy 412

E Eckold 92 Edridge- Green 308

Eisler 176 Eisner 191

Elschnig 190, 309, 384 van der Elst 318 El-Tarouti 311 Eppenstein 93 Erggelet 207, 213, 215, 
216 Esser 195, 208, 218

$\Gamma$ Fazakas 100, 103, 104,

109, no Federew 306 Federowa 306 Fehr 326 Feilchenfeld 200 Fejér 96, 106, no, 411 Ferree

306, 308 Fesüs 99, 100 Fialho 346, 388 Filatow 169 Finlay 185 Fischer 175, 182, 200,

$209,212,218,223$

324, 404, 415 Forsberg 224 Forti 322

Franceschetti 319 Fränkel 313 Freeman 314, 319 Frima 308 Frimann-Dahl 224 Fritz 310 Fuchs, A. 175 Fukamizu 184 Fulton 387 Funaishi 314 